http://dx.doi.org/10.12775/CLR.2015.006

\title{
DER SONDERIÜNDIGUNGSSCHUTZ BEHINDERTER ARBEITNEHMER IM DEUTSCHEN UND POLNISCHEN ARBEITSRECHT
}

\begin{abstract}
Zusammenfassung
Der deutsche Gesetzgeber hat die Zulässigkeit der Kündigung des Arbeitsverhältnisses mit schwerbehinderten Menschen und diesen gleichgestellten behinderten Menschen, die entsprechend in Abs. 2 und Abs. 3 des 2 SGB IX genannt werden, für den Arbeitgeber eingeschränkt. In den beiden Fällen ist eine vorherige Zustimmung des Integrationsamtes erforderlich. Der polnische Gesetzgeber hat sich im Gegensatz zum deutschen nicht dazu entschieden, solche Maßnahmen wie ein Verbot der Kündigung des Arbeitsverhältnisses wegen einer Behinderung, eine Verpflichtung des Arbeitgebers zur Einholung einer Zustimmung zur Entlassung des behinderten Arbeitnehmers oder einen geschlossenen Katalog der Kündigungsgründe im Fall von Menschen mit Behinderungen $z u$ beschließen. Er hat lediglich rechtliche Instrumente zur Stabilisierung der Beschäftigung von Menschen mit Behinderungen geschaffen, insbesondere die Pflicht zur Ausgliederung bzw. Einrichtung eines angemessenen Arbeitsplatzes mit dem dazugehörigen Sozialraum für den Arbeitnehmer, der infolge eines Arbeitsunfalls oder einer Berufskrankheit unfähig wurde, am bisherigen Arbeitsplatz zu arbeiten, und dessen Behinderung anerkannt wurde.
\end{abstract}

Keywords

Menschen mit Behinderungen - Arbeitsverhältnis - Diskriminierungsverbot - Besonderer Kündigungsschutz

\footnotetext{
* Marzena Szabłowska-Juckiewicz - Doktor der Rechtswissenschaften, wissenschaftliche Mitarbeiterin am Lehrstuhl für Arbeitsrecht der Fakultät für Recht und Verwaltung an der Nikolaus-Kopernikus-Universität Torun. Forschungsschwerpunkte: Schutz der Persönlichkeitsrechte, Schadensersatzhaftung des Arbeitgebers und Beschäftigung von Menschen mit Behinderungen.
} 


\section{EINLEITUNG}

Sowohl im deutschen als auch im polnischen Arbeitsrecht ist das Diskriminierungsverbot wegen einer Behinderung festgeschrieben. In Deutschland sind im Allgemeinen Gleichbehandlungsgesetz und in Polen im Arbeitsgesetzbuch Vorschriften enthalten, die zum Ziel haben, Benachteiligungen - auch in Bezug auf Entlassungsbedingungen aufgrund einer Behinderung $\mathrm{zu}$ verhindern oder $\mathrm{zu}$ beseitigen. Es steht außer Zweifel, dass niemand wegen einer Behinderung entlassen werden darf, es sei denn, unterschiedliche Behandlung ist rechtmäßig.

Der vorliegende Artikel betrifft den Kündigungsschutz. In der deutschen Literatur wird betont, dass „Diskriminierungs- und Kündigungsschutz durchaus nebeneinander bestehen können. Erster ist transaktionsbezogen, letzter gewährleistet Bestandsschutz. Einmal geht es um den Schutz vor Maßnahmen, die auf besonders schutzunwürdigen Motiven beruhen. Das andere Mal steht das Interesse des Arbeitnehmers an der Erhaltung seines Arbeitsplatzes im Vordergrund. Das Benachteiligungsverbot dagegen hat selbst im Beendigungszeitraum nicht den Bestand des Arbeitsverhältnisses im Auge“1.

Behinderte Menschen sollen am Arbeitsmarkt befördert werden. Um ihre Einstellungschancen zu verbessern, hat nicht nur der deutsche, sondern auch der polnische Gesetzgeber das System von Beschäftigungspflicht und Ausgleichsabgabe angenommen. Jedoch liegen im Bereich des Kündigungsschutzes behinderter Menschen wesentliche Unterschiede zwischen der deutschen und der polnischen Gesetzgebung vor: Die Regelungen in Polen setzen grundsätzlich keinen besonderen Kündigungsschutz ein.

Aus der Sicht der polnischen Arbeitsrechtslehre sind die deutschen Bestimmungen, die Arbeitsverhältnisse mit schwerbehinderten Menschen besonders schützen, sehr interessant. Es handelt sich um $\S \quad 85-92$ Sozialgesetzbuch (SGB) Neuntes Buch (IX) - Rehabilitation und Teilhabe behinderter Menschen vom 19. Juni 2001.

1 T. Leder, Das Diskriminierungsverbot wegen einer Behinderung. Schriften zum Sozialund Arbeitsrecht, Band 250, Berlin: Duncker \& Humblot 2006, S. 176. 


\section{Definitionen der Behinderung Und des MENSCHEN Mit BEHINDERUNG FÜR DIE ZWECKE DER BERUFLICHEN REHABILITATION UND DER BESCHÄFTIGUNG}

\subsection{AllGemeine BemerkUnGeN}

In der Präambel (e) des Übereinkommens der Vereinten Nationen vom 13. Dezember 2006 über die Rechte von Menschen mit Behinderungen (im Folgenden: UN-Konvention) wird darauf hingewiesen, dass das Verständnis von Behinderung sich ständig weiterentwickelt. Im Lichte der UN-Konvention gelten Menschen, die langfristige körperliche, seelische, geistige oder Sinnesbeeinträchtigungen haben, welche sie in Wechselwirkung mit verschiedenen Barrieren an der vollen, wirksamen und gleichberechtigten Teilhabe an der Gesellschaft hindern können, als Menschen mit Behinderungen (Artikel 1 Satz 2). Die UN-Konvention wurde von der Europäischen Union ratifiziert ${ }^{2}$. Sie wurde von allen Mitgliedstaaten der Europäischen Union unterzeichnet und von den meisten, einschließlich Deutschland ${ }^{3}$ und Polen ${ }^{4}$, ratifiziert. In der Mitteilung der Kommission an das Europäische Parlament, den Rat, den Europäischen Wirtschafts- und Sozialausschuss und den Ausschuss der Regionen vom 15. November 2010 „Europäische Strategie zugunsten von Menschen mit Behinderungen 2010-2020: Erneuertes Engagement für ein barrierefreies Europa " 5 wird darauf verwiesen, dass die UN-Konvention - das erste rechtlich bindende Rechtsinstrument im Bereich der Menschenrechte, bei dem die Europäische Union und ihre Mitgliedstaaten Vertragsparteien sind - bald in der gesamten Europäischen Union gelten wird. In der einschlägigen Fachliteratur wird betont, dass für die rechtmäßige und wirksame Umsetzung der gesamten UNKonvention eine Anpassung der im EU- und im nationalen Recht

\footnotetext{
2 Beschluss 2010/48/EG des Rates vom 16.11.2009 über den Abschluss des Übereinkommens der Vereinten Nationen über die Rechte von Menschen mit Behinderungen durch die Europäische Gemeinschaft (ABl. L 23, S. 35).

3 Geschehen am 24.02.2009.

4 Die UN-Konvention wurde vom Präsidenten der Republik Polen am 6.09.2012 ratifiziert, Dz.U. [Gesetzblatt] 2012, Pos. 1169.

$5 \operatorname{KOM}(2010) 636$.
} 
vorhandenen Definitionen an die Vorgaben der EU-Konvention zwingend notwendig ist. Außerdem muss das EU- und nationale Recht in Übereinstimmung mit der UN-Konvention ausgelegt werden'.

$\mathrm{Zu}$ beachten ist, dass die Richtlinie des Rates 2000/78/EG vom 27. November 2000 zur Festlegung eines allgemeinen Rahmens für die Verwirklichung der Gleichbehandlung in Beschäftigung und Beruf ${ }^{7}$ keine Definition der Behinderung und des behinderten Menschen für die Zwecke von Beschäftigung und Beruf beinhaltet. Der Gerichtshof der Europäischen Union stellt im Urteil vom 11. Juli 2005 fest, dass im Hinblick auf den sachlichen Anwendungsbereich der Richtlinie 2000/78 „der Begriff Behinderung so zu verstehen ist, dass er eine Einschränkung erfasst, die insbesondere auf körperliche, geistige oder psychische Beeinträchtigungen zurückzuführen ist und die ein Hindernis für die Teilhabe des Betreffenden am Berufsleben bildet" Der Gerichtshof steht auf dem Standpunkt, „dass die Bedeutung, die der Gemeinschaftsgesetzgeber den Maßnahmen zur Einrichtung des Arbeitsplatzes nach Maßgabe der Behinderung beigemessen hat, zeigt, dass er an Fälle gedacht hat, in denen die Teilhabe am Berufsleben über einen langen Zeitraum eingeschränkt ist. Damit die Einschränkung unter den Begriff Behinderung fällt, muss daher wahrscheinlich sein, dass sie von langer Dauer ist" . $^{\text {. }}$

Im nationalen Recht der Mitgliedstaaten der Europäischen Union, einschließlich des deutschen und polnischen Rechts, werden Definitionen der Behinderung und des behinderten Menschen für die Zwecke der beruflichen Rehabilitation und der Beschäftigung gebildet. Diese weisen gewisse Unterschiede auf. Von der Definition der Begriffe „Behinderung“ und „behinderter Mensch“ hängt der persönliche

\footnotetext{
6 J. Kapelańska-Pręgowska, Wdrażanie, stosowanie i kontrola wykonywania Konwencji ONZ o prawach osób niepetnosprawnych [Umsetzung, Anwendung und Kontrolle der Umsetzung des UN-Übereinkommens über die Rechte von Menschen mit Behinderungen], [in:] A. GiedrewiczNiewińska, M. Szabłowska-Juckiewicz (Red.), Zatrudnianie osób niepetnosprawnych. Regulacje prawne [Beschäftigung von behinderten Menschen. Rechtliche Regelungen], Warszawa: Difin 2014, S. 35 .

7 ABl. L 303, S. 16, im Folgenden: Richtlinie 2000/78.

8 Urteil des Gerichtshofs der Europäischen Union vom 11.07.2005 in der Rechtssache C-13/05 Sonia Chacón Navas v. Eurest Colectividades SA, Pkt. 43 und Pkt. 45, Lex Nr. 226783.
} 
Anwendungsbereich des Kündigungsschutzes, der den behinderten Menschen gewährt wird.

\subsection{DEFINITIONEN IM DEUTSCHEN RECHT}

Der deutsche Gesetzgeber formuliert die Definition des behinderten Menschen in § 2 Sozialgesetzbuch (SGB) IX vom 19. Juni 20019. Gemäß $\S 2$ Abs. 1 SGB IX sind Menschen behindert, wenn ihre körperliche Funktion, geistige Fähigkeit oder seelische Gesundheit mit hoher Wahrscheinlichkeit länger als sechs Monate von dem für das Lebensalter typischen Zustand abweichen und daher ihre Teilhabe am Leben in der Gesellschaft beeinträchtigt ist. Sie sind von Behinderung bedroht, wenn die Beeinträchtigung $\mathrm{zu}$ erwarten ist. In der Fachliteratur wird darauf hingewiesen, dass "die Definition körperliche, geistige und seelische Abweichungen von dem für das Lebensalter typischen Zustand gleichgewichtig einbezieht. Art und Ursache der Behinderung sind nicht mehr maßgeblich"10. Maßgeblich ist dagegen die Dauer der körperlichen, geistigen und seelischen Abweichungen. Als behinderte Menschen gelten nur diejenigen, bei denen diese Abweichungen länger als sechs Monate dauern.

„Im deutschen Recht waren die körperlichen, geistigen und seelischen Behinderungen nach wie vor in der Tabelle Grad der Behinderung/Minderung der Erwerbsfähigkeit zusammengefasst, die als Anhaltspunkt für die ärztliche Gutachtertätigkeit im sozialen Entschädigungsrecht und nach dem Teil 2 des SGB IX vom Bundesministerium für Arbeit und Soziales im Jahre 2008 zuletzt herausgegeben wurde" ${ }^{\prime 1}$.

Auf der Grundlage der Vorschriften des Sozialgesetzbuches IX wurden die Kategorien "schwerbehinderte Menschen" und diesen "gleichgestellte behinderte Menschen" gebildet und ihr Bedeutungsumfang bestimmt. Gemäß § 2 Abs. 2 SGB IX sind Menschen schwerbehindert, wenn bei ihnen

9 BGBl. I S. 1046 m. Änd., im Folgenden: SGB IX.

10 D. Neumann, [in:] D. Neumann, R. Pahlen, M. Majerski-Pahlen, Sozialgesetzbuch IX - Rehabilitation und Teilhabe behinderter Menschen. Kommentar, 12. Auflage, München: C.H. Beck 2010, S. 16.

11 Ibidem, S. 17-18. 
ein Grad der Behinderung von wenigstens 50 vorliegt und sie ihren Wohnsitz, ihren gewöhnlichen Aufenthalt oder ihre Beschäftigung auf einem Arbeitsplatz im Sinne des § 73 rechtmäßig im Geltungsbereich dieses Gesetzbuches haben. \& 2 Abs. 3 SGB IX „regelt dagegen die Voraussetzungen für die Gleichstellung mit schwerbehinderten Menschen"12. Nach dieser Vorschrift sollen schwerbehinderten Menschen behinderte Menschen mit einem Grad der Behinderung von weniger als 50, aber wenigstens 30 gleichgestellt werden, bei denen die übrigen Voraussetzungen des Absatzes 2 vorliegen, wenn sie infolge ihrer Behinderung ohne die Gleichstellung einen geeigneten Arbeitsplatz im Sinne des \& 73 nicht erlangen oder nicht behalten können. Ob jemand als "schwerbehinderter Mensch" oder "gleichgestellter behinderter Mensch" anerkannt wird, hängt von der Feststellung des Behinderungsgrades sowie davon $a b$, ob der Betreffende seinen festen Wohnsitz, seinen gewöhnlichen Aufenthalt oder seinen Arbeitsplatz in Deutschland hat. Eine Prämisse für die Gleichstellung mit schwerbehinderten Menschen ist außerdem der Umstand, dass ohne diese Gleichstellung der Betreffende keine Arbeitsstelle finden bzw. behalten könnte. Das Verfahren zur Gleichstellung richtet sich nach § 68 Abs. 2 SGB IX, die Feststellung des Grades der Behinderung nach $\S 69$ SGB IX13.

\subsection{DEFINITIONEN IM POLNISCHEN RECHT}

Im polnischen Recht sind die Begriffe "Behinderung“ und „behinderter Mensch“ für die Zwecke der beruflichen Rehabilitation und der Beschäftigung im Gesetz über berufliche und soziale Rehabilitation sowie Beschäftigung von Menschen mit Behinderungen vom 27. August 1997 enthalten ${ }^{14}$.

Nach Artikel 2 Pkt. 10 des Rehabilitationsgesetzes bezeichnet Behinderung eine dauerhafte oder vorübergehende Unfähigkeit eines Menschen, seine sozialen Rollen zu erfüllen, die auf eine dauerhafte

\footnotetext{
12 Ibidem, S. 27.

13 Ibidem.

14 Einheitlicher Text: Dz.U. [Gesetzblatt] 2011, Nr. 127, Pos. 721 m. Änd., im Folgenden: Rehabilitationsgesetz.
} 
oder vorübergehende Beeinträchtigung seiner Körperfunktionen zurückzuführen ist und insbesondere eine Erwerbsminderung verursacht. Der polnische Gesetzgeber unterscheidet im Kontext der Beschäftigung und der beruflichen Rehabilitation zwischen drei Behinderungsgraden: erheblich, mäßig und leicht (Artikel 3 Abs. 1 Rehabilitationsgesetz).

In der einschlägigen Fachliteratur wird kritisch beurteilt, dass in der Definition der Behinderung im Kontext der Beschäftigung der Begriff „Erwerbsminderung“ hervorgehoben wurde15. Der Gesetzgeber sieht die Ursachen für die Unfähigkeit eines Menschen, seine sozialen Rollen zu erfüllen, in seinem Gesundheitszustand („Unfähigkeit, die sozialen Rollen $\mathrm{zu}$ erfüllen, die auf eine dauerhafte oder vorübergehende Beeinträchtigung der Körperfunktionen zurückzuführen ist"), übersieht aber die Barrieren der Umwelt, die dem behinderten Menschen eine Beschäftigung erschweren oder gar unmöglich machen können. Somit hängt er dem medizinischen Behinderungsbegriff an. „Der Übergang vom medizinischen zum sozialen Behinderungsbegriff (...) hat im polnischen Recht nach wie vor nicht stattgefunden"16. Außerdem ist zu bemerken, dass nicht jede Beeinträchtigung der Körperfunktionen, die zur Erfüllung der sozialen Rollen unfähig macht, als Behinderung angesehen werden kann. Eine Behinderung liegt nämlich nur dann vor, wenn die Beeinträchtigung der Körperfunktionen bleibend oder dauerhaft ist. Bei Personen, die das 16. Lebensjahr nicht vollendet haben, muss die körperlich bzw. geistige Beeinträchtigung länger als 12 Monate dauern (Artikel 4a Abs. 1 Rehabilitationsgesetz). Bei Personen dagegen, die das 16. Lebensjahr vollendet haben, hat der Gesetzgeber nicht festgelegt, wie lange die Beeinträchtigung dauern muss, damit sie einem der drei Behinderungsgrade zugerechnet werden kann.

Damit jemand als Mensch mit Behinderung anerkannt wird, reicht es nicht aus, dass eine tatsächliche Behinderung im Sinne des Gesetzes über

\footnotetext{
15 A. Tyśkiewicz-Mazur, Definicje niepetnosprawności na potrzeby rehabilitacji zawodowej $i$ zatrudnienia [Definitionen der Behinderung für die Zwecke der beruflichen Rehabilitation und der Beschäftigung], [in:] A. Giedrewicz-Niewińska, M. Szabłowska-Juckiewicz (Red.), Zatrudnianie osób niepetnosprawnych. Regulacje prawne [Beschäftigung von behinderten Menschen. Rechtliche Regelungen], Warszawa: Difin 2014, S. 45. Ähnlich: A. Żak, Prawne pojęcie niepetnosprawności [Der rechtliche Behinderungsbegriff], Studia z zakresu prawa pracy i polityki społecznej [Studien zum Arbeitsrecht und zur Sozialpolitik] 2003/2004, S. 355.

16 Tyśkiewicz-Mazur, Fußnote 16, S. 39.
} 
berufliche und soziale Rehabilitation sowie Beschäftigung von Menschen mit Behinderungen vorhanden ist. In Artikel 1 des Rehabilitationsgesetzes ist eine formale Definition des Menschen mit Behinderung enthalten ${ }^{17}$. Danach gelten nur diejenigen als Menschen mit Behinderungen, deren Behinderung in einem entsprechenden Bescheid festgestellt wurde. Es handelt sich um die Feststellung eines der drei Grade der Behinderung, Feststellung einer vollen oder teilweisen Erwerbsminderung nach Maßgabe des Artikel 12 des Gesetzes über Renten aus dem Sozialversicherungsfonds vom 17. Dezember 199818 oder eine Feststellung der Behinderung vor der Vollendung des 16. Lebensjahres.

Zuständig für die Feststellung der Behinderung und des Grades der Behinderung sind in erster Instanz die Kreisausschüsse für Behinderungsfeststellung sowie in zweiter Instanz die Woiwodschaftsausschüsse für Behinderungsfeststellung (Artikel 6 Abs. 1 Rehabilitationsgesetz). Bei Personen unter dem 16. Lebensjahr wird eine Behinderung festgestellt, während bei Personen, die das 16. Lebensjahr vollendet haben, muss die Feststellung eines der drei Behinderungsgrade herbeigeführt werden ${ }^{19}$. Den Feststellungen eines Behinderungsgrades sind Bescheide der ärztlichen Gutachter der Sozialversicherungsanstalt [Zakład Ubezpieczeń Społecznych] gleichwertig, die nach dem SVF-Rentengesetz erteilt werden: Der Bescheid über die volle Erwerbsminderung und Unfähigkeit zur selbständigen Lebensführung ist der Feststellung eines erheblichen Behinderungsgrades, der Bescheid über eine volle Erwerbsminderung ist der Feststellung eines mäßigen Behinderungsgrades und der Bescheid über eine teilweise Erwerbsunfähigkeit ist der Feststellung eines leichten Behinderungsgrades gleichwertig (Artikel 5 Rehabilitationsgesetz).

Es ist festzustellen, dass im Hinblick auf die Gleichstellung der Bescheide über die volle bzw. teilweise Erwerbsminderung mit

\footnotetext{
17 Vgl. Żak, Fußnote 16, S. 349.

18 Einheitlicher Text: Dz.U. [Gesetzblatt] von 2013, Pos. 1440 m. Änd., im Folgenden: SVF-Rentengesetz.

19 Mehr dazu s. I. Radziwon, Orzekanie o niepetnosprawności oraz o jej stopniu [Verfahren zur Feststellung der Behinderung und ihres Grades], [in:] A. Giedrewicz-Niewińska, M. Szabłowska-Juckiewicz (Red.), Zatrudnianie osób niepetnosprawnych. Regulacje prawne [Beschäftigung von behinderten Menschen. Rechtliche Regelungen], Warszawa: Difin 2014, S. 50-52.
} 
den Feststellungen des Behinderungsgrades sowie auf die Anerkennung von Personen als behinderte Menschen, denen ein ärztlicher Gutachter der Sozialversicherungsanstalt einen Bescheid über die volle bzw. teilweise Erwerbsminderung erteilt hat, Artikel 40 des Arbeitsgesetzbuches Bedenken wecken kann. Dort wird nämlich vorgeschrieben, dass Artikel 39 des Arbeitsgesetzbuches nicht angewendet wird, wenn der Arbeitnehmer eine Rente wegen voller Erwerbsminderung erworben hat. Der besondere Kündigungsschutz für Personen im sog. Vorruhestandsalter erstreckt sich nicht auf Arbeitnehmer, denen volle Erwerbsminderung attestiert wurde und die aus diesem Grund einen Rentenanspruch erworben haben. Im Lichte des Artikel 5 des Rehabilitationsgesetzes ist der Bescheid über die volle Erwerbsminderung der Feststellung eines mäßigen Behinderungsgrades gleichwertig. Folglich kommt es vor dem Hintergrund des Artikel 40 des Arbeitsgesetzbuches $\mathrm{zu}$ einer unterschiedlichen Position von Personen, die aufgrund eines vom ärztlichen Gutachter der Sozialversicherungsanstalt erteilten Bescheids über die volle bzw. teilweise Erwerbsminderung als behinderte Menschen anerkannt wurden und denjenigen, denen der mäßige Behinderungsgrad vom Kreis- bzw. Woiwodschaftsausschuss für Behinderungsfeststellung attestiert wurde. Der besondere Kündigungsschutz für Personen im sog. Vorruhestandsalter wurde nur hinsichtlich der Personen ausgeschlossen, bei denen aufgrund des entsprechenden Bescheides des ärztlichen Gutachters der Sozialversicherungsanstalt der mäßige Behinderungsgrad bestätigt wurde. Offenkundig wäre es zweckmäßig, die Gleichsetzung der Bescheide über die volle bzw. teilweise Erwerbsminderung mit den Feststellungen eines Behinderungsgrades abzuschaffen. Eine solche Änderung würde Zweifel in Bezug auf Artikel 40 des Arbeitsgesetzbuches beseitigen und zudem mehr Klarheit bei der Definition des behinderten Menschen schaffen, so dass behinderte Menschen nicht mehr mit voll bzw. teilweise Erwerbsgeminderten gleichgesetzt würden. 


\section{Verbot der DisiKriminieRUNG BEI DER BEENDIGUNG DES ARBEITSVERHÄLTNISSES UND VERPFLICHTUNG ZU RATIONALEN ANPASSUNGEN ALS INSTRUMENTE ZUR STABILISIERUNG DER BESCHÄFTIGUNG}

\subsection{Allgemeine BEMERKUNGEN}

Das Verbot der Diskriminierung wegen einer Behinderung bei der Beendigung des Arbeitsverhältnisses ist als ein Instrument zur Stabilisierung der Beschäftigung aller Menschen mit Behinderungen anzusehen, ungeachtet des Zeitpunkts, an dem die Behinderung eingetreten ist, bzw. der Art und des Grades der Behinderung. Die Einführung dieses Verbots ermöglicht die Verwirklichung des Rechts auf Arbeit insbesondere für Menschen, deren Behinderung während des Beschäftigungszeitraums eingetreten ist. Der Stabilisierung der Beschäftigung behinderter Menschen dient außerdem die Verpflichtung des Arbeitgebers, rationale Anpassungen an die Bedürfnisse der Menschen mit Behinderungen im Betrieb vorzunehmen.

Auf die Gesamtheit der im deutschen und polnischen Recht geltenden Regelungen zur Gleichbehandlung in Beschäftigung und Beruf hatten die EU-Vorgaben einen wesentlichen Einfluss. Daher erscheint es als angebracht, die Regelungen der Europäischen Union zur Diskriminierung wegen einer Behinderung bei der Beendigung des Arbeitsverhältnisses sowie zu den Anpassungen an die Bedürfnisse behinderter Menschen im Betrieb summarisch darzustellen.

Im Unionsrecht wurde der allgemeine Rahmen für die Bekämpfung der Diskriminierung in Beschäftigung und Beruf wegen einer Behinderung mit der Richtlinie 2000/78 geschaffen. Der EU-Gesetzgeber formuliert das Verbot jeglicher Diskriminierung, darunter der unmittelbaren oder mittelbaren Diskriminierung wegen einer Behinderung, der Belästigung wegen einer Behinderung, und der Anweisung zur Diskriminierung einer Person wegen einer Behinderung ${ }^{20}$.

20 S. Präambel (12) und Artikel 2 der Richtlinie 2000/78. 
Die Richtlinie 2000/78 findet insbesondere auf die Entlassungsbedingungen Anwendung (Artikel 3 Abs. 1 Buchst. c). Unter Bezug auf die Bestimmungen der vorgenannten Richtlinie führt der Gerichtshof der Europäischen Union aus, dass „das Verbot der Diskriminierung wegen einer Behinderung bei Entlassungen, (...) der Entlassung wegen einer Behinderung entgegensteht, die unter Berücksichtigung der Verpflichtung, angemessene Vorkehrungen für Menschen mit Behinderungen zu treffen, nicht dadurch gerechtfertigt ist, dass die betreffende Person für die Erfüllung der wesentlichen Funktionen ihres Arbeitsplatzes nicht kompetent, fähig oder verfügbar ist". Der Gerichtshof weist jedoch darauf hin, dass „eine Person, der von ihrem Arbeitgeber ausschließlich wegen Krankheit gekündigt worden ist, nicht von dem durch die Richtlinie 2000/78 zur Bekämpfung der Diskriminierung wegen einer Behinderung geschaffenen allgemeinen Rahmen erfasst wird“. Nach Auffassung des Gerichtshofs "hat der Gesetzgeber mit der Verwendung des Begriffes «Behinderung» (...) bewusst ein Wort gewählt, das sich von dem der «Krankheit» unterscheidet. Daher lassen sich die beiden Begriffe nicht schlicht und einfach einander gleichsetzen“. Der Gerichtshof betont, dass „Krankheit als solche nicht als ein weiterer Grund angesehen werden kann, derentwegen Personen zu diskriminieren nach der Richtlinie 2000/78 verboten ist" ${ }^{\prime 21}$.

Der EU-Gesetzgeber lässt eine unterschiedliche Behandlung in Beschäftigung und Beruf aus den in Artikel 1 der Richtlinie 2000/78 genannten Gründen, darunter wegen einer Behinderung, unter bestimmten Umständen zu. Gem. Artikel 4 Abs. 1 der Richtlinie 2000/78 können die Mitgliedstaaten entscheiden, dass eine Ungleichbehandlung wegen eines Merkmals, das im Zusammenhang mit einem der in Artikel 1 genannten Diskriminierungsgründe steht, keine Diskriminierung darstellt, wenn das betreffende Merkmal aufgrund der Art einer bestimmten beruflichen Tätigkeit oder der Bedingungen ihrer Ausübung eine wesentliche und entscheidende berufliche Anforderung darstellt, sofern es sich um einen rechtmäßigen Zweck und eine angemessene Anforderung handelt.

21 Urteil des Gerichtshofs der Europäischen Union, Fußnote 9, Pkt. 51-52, Pkt. 44 und 57. 
In der Richtlinie 2000/78 wird darüber hinaus auf „die Notwendigkeit hingewiesen, behinderten Menschen ausgleichende Sonderrechte zu gewähren“22. In der Präambel der Richtlinie werden Maßnahmen, die darauf abstellen, den Bedürfnissen von Menschen mit Behinderungen am Arbeitsplatz Rechnung zu tragen, als das wichtigste Element der Bekämpfung von Diskriminierungen wegen einer Behinderung betrachtet. Es handelt sich z.B. um eine entsprechende Gestaltung der Räumlichkeiten, eine Anpassung des Arbeitsgeräts, des Arbeitsrhythmus, der Aufgabenverteilung oder des Angebots an Ausbildungs- und Einarbeitungsmaßnahmen (Pkt. 16 und Pkt. 20). Der EU-Gesetzgeber formuliert die Verpflichtungen des Arbeitgebers, angemessene Vorkehrungen für Menschen mit Behinderungen zu treffen, und betont, dass die Mitgliedstaaten entsprechende Maßnahmen ergreifen müssen, um die Beschäftigungschancen der behinderten Menschen auszugleichen (sog. Positive Maßnahmen) ${ }^{23}$.

\subsection{REGELUNGEN IM DEUTSCHEN RECHT}

Der Diskriminierung vor allem wegen Behinderungen entgegenzuwirken und diese zu bekämpfen ist das Ziel des Allgemeinen Gleichbehandlungsgesetzes ${ }^{24}$, das insbesondere die Richtlinie 2000/78 implementiert ${ }^{25}$. Der deutsche Gesetzgeber vertritt ähnlich dem EUGesetzgeber die Auffassung, dass eine Diskriminierung wegen Behinderungen in Bezug auf Entlassungsbedingungen unzulässig ist (§ 2 Abs. 1 (2) AGG).

Der Inhalt des $\S 2$ Abs. 4 AGG ist nicht zu übersehen. Nach dieser Vorschrift gelten für Kündigungen ausschließlich die Bestimmungen zum allgemeinen und besonderen Kündigungsschutz. Das Bundesarbeitsgericht betont im Urteil vom 17. Oktober 2013 (8 AZR 742/12), dass „es umstritten

\footnotetext{
22 M. Szabłowska, [in:] A. Wróbel (Red.), Karta Praw Podstawowych Unii Europejskiej. Komentarz [Charta der Grundrechte der Europäischen Union. Kommentar], Warszawa: C.H. Beck 2013, S. 848.

23 S. Artikel 5 und Artikel 7 Abs. 2 der Richtlinie 2000/78.

24 Gesetz zur Umsetzung europäischer Richtlinie zur Verwirklichung des Grundsatzes der Gleichbehandlung vom 14.08.2006, BGBl. 2006, Nr. 39, S. 1876 m. Änd., im Folgenden: AGG.

25 Mehr dazu s. A. Schleusener, [in:] A. Schleusener, J. Suckow, B. Voigt, AGG. Kommentar zum Allgemeinen Gleichbehandlungsgesetz, Neuwied: Luchterhand Fachverlag 2007, S. 23-24.
} 
ist, welche Bedeutung § 2 Abs. 4 AGG im Einzelnen zukommt (...). Jedenfalls sind die Diskriminierungsverbote des AGG einschließlich der im Gesetz vorgesehenen Rechtfertigungen für unterschiedliche Behandlungen bei der Auslegung der unbestimmten Rechtsbegriffe des Kündigungsschutzgesetzes in der Weise $\mathrm{zu}$ beachten, als sie Konkretisierungen des Sozialwidrigkeitsbegriffs darstellen. Verstößt eine ordentliche Kündigung gegen Benachteiligungsverbote des AGG (§§ 1 bis 10 AGG), so kann dies zur Sozialwidrigkeit der Kündigung nach $\S 1$ KSchG führen". Folglich entfaltet die ordentliche Kündigung keine Rechtswirkung, sofern sie gesellschaftlich und sozial unbegründet ist und einen Arbeitnehmer betrifft, der in demselben Betrieb ununterbrochen seit länger als 6 Monaten beschäftigt ist.

Das Bundesarbeitsgericht nahm $\mathrm{zu}$ den Rechtswirkungen der Diskriminierung im Zusammenhang mit der Beendigung des Arbeitsverhältnisses mehrfach Stellung. Im Urteil vom 19. Dezember 2013 (6 AZR 190/12) befand es, dass „eine ordentliche Kündigung, die einen Arbeitnehmer, auf den das Kündigungsschutzgesetz (noch) keine Anwendung findet, aus einem der in § 1 AGG genannten Gründe diskriminiert, (...) unwirksam ist". Im Urteil vom 12. Dezember 2013 (8 AZR 838/12) stellte es dagegen fest, dass „bei diskriminierenden Kündigungen unbeschadet des § 2 Abs. 4 AGG ein Anspruch auf den Ersatz immaterieller Schäden nach § 15 Abs. 2 AGG grundsätzlich möglich ist".

\subsection{REGELUNGEN IM POLNISCHEN RECHT}

Die EU-Normen beeinflussten ebenfalls die Regelungen, die vom polnischen Gesetzgeber in das Arbeitsgesetzbuch aufgenommen wurden. „Die Vorschriften des Arbeitsgesetzbuches zur Gleichbehandlung in der Beschäftigung stützen sich großenteils auf die Vorgaben der EU-Richtlinien (...) und die umfangreiche Rechtsprechung des Gerichtshofs der Europäischen Union zu diesen Richtlinien“26.

Nach Artikel 183a $\S 2$ des Arbeitsgesetzbuches bedeutet Gleichbehandlung bei der Beschäftigung, dass keine Diskriminierung

26 L. Florek, Prawo pracy [Arbeitsrecht], Warszawa: C.H. Beck 2012, S. 17. 
aus den in $\S 1$ genannten Gründen stattfindet. Der polnische Gesetzgeber führt ähnlich dem EU-Gesetzgeber das Verbot einer unmittelbaren und mittelbaren Diskriminierung, einer Belästigung bei der Beschäftigung und der Handlungen, die andere Personen dazu anregen, den Gleichbehandlungsgrundsatz bei der Beschäftigung zu verletzen, bzw. sie zur Verletzung dieses Grundsatzes anweisen.

Behinderung zählt zu den Eigenschaften, die dem Diskriminierungsverbot unterliegen. Im Arbeitsgesetzbuch wird der sachliche Anwendungsbereich des Diskriminierungsverbotes bei der Beschäftigung weit ausgelegt, was mit dem EU-Recht im Einklang steht ${ }^{27}$. Dieses Verbot gilt insbesondere für die Beendigung des Arbeitsverhältnisses. Nach Maßgabe des Artikel 183a $§ \quad 1$ des Arbeitsgesetzbuches sind Arbeitnehmer bei der Beendigung des Arbeitsverhältnisses gleich zu behandeln, darunter auch unabhängig von etwaiger Behinderung. In der einschlägigen Fachliteratur wird hervorgehoben, dass angesichts dieser Vorschrift „die Behinderung eines Arbeitnehmers (...) keinen Grund für die Kündigung bzw. z.B. für die Auswahl des Arbeitnehmers bei Massenentlassungen darstellen darf" 28 .

Unter dem Einfluss des EU-Rechts wurde außerdem Artikel 23a ins Gesetz über berufliche und soziale Rehabilitation sowie Beschäftigung von Menschen mit Behinderungen vom 27. August 1997 eingebaut ${ }^{29}$. In Artikel 23a des Rehabilitationsgesetzes wurden die Pflichten des Arbeitgebers festgeschrieben, rationale Anpassungen an die Bedürfnisse der Menschen mit Behinderungen im Betrieb vorzunehmen. Es ist hervorzuheben, dass die Unterlassung rationaler Anpassungen an die Bedürfnisse der Menschen mit Behinderungen als Verletzung des Gleichbehandlungsgrundsatzes bei der Beschäftigung im Sinne

\footnotetext{
27 Vgl. L. Mitrus, Wptyw regulacji wspólnotowych na polskie prawo pracy [Einfluss der Gemeinschaftsregelungen auf das polnische Arbeitsrecht], Kraków: Kantor Wydawniczy Zakamycze 2006, S. 193 und S. 195.

28 M. Latos-Miłkowska, Ochrona trwałości stosunku pracy pracowników niepetnosprawnych $i$ opiekunów osób niepetnosprawnych [Kündigungsschutz bei behinderten Arbeitnehmern und Behindertenbetreuern], [in:] A. Giedrewicz-Niewińska, M. Szabłowska-Juckiewicz (Red.), Zatrudnianie osób niepetnosprawnych. Regulacje prawne [Beschäftigung von behinderten Menschen. Rechtliche Regelungen], Warszawa: Difin 2014, S. 258.

29 Einheitlicher Text: Dz.U. [Gesetzblatt] von 2011, Nr. 127, Pos. 721 m. Änd.
} 
des Arbeitsgesetzbuches eingestuft wird (Artikel 23a Abs. 3 Rehabilitationsgesetz).

\section{BESONDERER KÜNDIGUNGSSCHUTZ FÜR MENSCHEN MIT BEHINDERUNGEN IM DEUTSCHEN RECHT}

\subsection{ALlGemeine BEMERKUnGeN}

Der besondere Kündigungsschutz für Menschen mit Behinderungen wird im deutschen Recht in den $\S \S 85-92$ [SGB IX] geregelt. „Die §§ 85-92 [SGB IX] stehen mit der gesamten Rechtsordnung, insbesondere den Grundrechten, den weiteren Regelungen des SGB IX und dem allgemeinem Kündigungsschutz in Wechselwirkung. Die jeweiligen Rechtssätze müssen daraufhin befragt werden, ob und auf welche Weise sie auf den Sonderkündigungsschutz Einfluss nehmen“30. Bei diesen Vorschriften handelt es sich um zwingendes Recht. Vereinbarungen des Arbeitsvertrages, in denen der schwerbehinderte Arbeitnehmer auf den Sonderkündigungsschutz verzichtet, sind unwirksam ${ }^{31}$.

Der besondere Kündigungsschutz erstreckt sich auf schwerbehinderte und diesen gleichgestellte behinderte Menschen, die entsprechend in Abs. 2 und Abs. 3 des $\S 2$ SGB IX genannt werden. Der deutsche Gesetzgeber hat bei diesen Arbeitnehmern die Zulässigkeit der Kündigung des Arbeitsverhältnisses sowie der Beendigung des Arbeitsverhältnisses ohne Kündigung eingeschränkt. In den beiden Fällen ist eine vorherige Zustimmung des Integrationsamtes erforderlich ${ }^{32}$. Bei dieser Zustimmung handelt es sich um einen öffentlich-rechtlichen Verwaltungsakt, der für die Wirksamkeit der Kündigung bzw. der Beendigung des Arbeitsverhältnisses durch den Arbeitgeber ohne Kündigung vorausgesetzt wird. Eine Erklärung über die Kündigung bzw. die Beendigung des Arbeitsverhältnisses ohne Kündigung, die dem behinderten Arbeitnehmer ohne vorherige Zustimmung des

30 Ch. Lampe, Der Kündigungsschutz behinderter Arbeitnehmer (§§ 85-92 SGB IX). Leitfaden für die Praxis, Köln: Luchterhand 2009, S. 2.

31 N. Besgen, Schwerbehindertenrecht. Arbeitsrechtliche Besonderheiten, Berlin: Erich Schmidt Verlag 2009, S. 87.

32 S. § 85 und § 91 SGB IX. 
Integrationsamtes zugestellt wird, ist nach Maßgabe des § 234 BGB unwirksam ${ }^{33}$.

Darüber hinaus wird der Schutz schwerbehinderter Menschen durch die Mindestküngigungsfrist verwirklicht. Die Kündigungsfrist beträgt mindestens vier Wochen (§ 86 SGB IX).

Der Sonderkündigungsschutz gilt nicht für schwerbehinderte Menschen, deren Arbeitsverhältnis zum Zeitpunkt des Zugangs der Kündigungserklärung ohne Unterbrechung noch nicht länger als sechs Monate besteht oder die auf Stellen im Sinne des $\S 73$ Abs. 2 Nr. 2 bis 5 beschäftigt werden oder deren Arbeitsverhältnis durch Kündigung beendet wird, sofern sie das 58. Lebensjahr vollendet haben und Anspruch auf eine Abfindung, Entschädigung oder ähnliche Leistung auf Grund eines Sozialplanes haben oder Anspruch auf Knappschaftsausgleichsleistung nach dem Sechsten Buch oder auf Anpassungsgeld für entlassene Arbeitnehmer des Bergbaus haben, wenn der Arbeitgeber ihnen die Kündigungsabsicht rechtzeitig mitgeteilt hat und sie der beabsichtigten Kündigung bis zu deren Ausspruch nicht widersprechen (§ 90 SGB IX).

In der Fachliteratur wird darauf hingewiesen, dass der Kündigungsschutz für schwerbehinderte Menschen in allen Betrieben, unabhängig von der Beschäftigtenzahl gilt ${ }^{34}$.

\subsection{EINSCHRÄNKUNGEN BEI DER ZULÄSSIGKEIT DER KÜNDIGUNG DURCH DEN ARBEITGEBER}

Die Kündigung des Arbeitsverhältnisses mit schwerbehinderten Menschen durch den Arbeitgeber bedarf der vorherigen Zustimmung des Integrationsamtes (§ 85 SGB IX). Die Zustimmung zur Kündigung beantragt der Arbeitgeber bei dem für den Sitz des Betriebes oder Dienststelle zuständigen Integrationsamt schriftlich (§ 87 Abs. 1 Satz 1 SGB IX). In der Fachliteratur wird hervorgehoben, dass „nur der Arbeitgeber selbst beziehungsweise die von ihm hierzu bevollmächtigte Person (Betriebsleiter, Personalsachbearbeiter)

33 Neumann, Fußnote 11, S. 341-342.

34 Besgen, Fußnote 32, S. 57. 
oder das für ihn satzungs- oder gesellschaftsrechtlich handelnde Organ (Geschäftsführer, Vorstandsmitglied) antragsbefugt ist“" 35 .

Der Antrag auf Zustimmung zur Kündigung bedarf der schriftlichen Form „und muss demgemäß nach $\S 126$ Abs. 1 BGB unterschrieben werden. (...) Zum Antrag gehören deshalb notwendig folgende Daten: Name und (wegen § 87 Abs. 2) Adresse des schwerbehinderten Menschen, Art und bisherige Dauer des Arbeitsverhältnisses und der Tätigkeit, Name, Art und Sitz des Betriebs, beabsichtigter Kündigungstermin und Endzeitpunkt des Arbeitsverhältnisses“36.

Das Integrationsamt soll die Entscheidung, falls erforderlich auf Grund mündlicher Verhandlung, innerhalb eines Monats vom Tage des Eingangs des Antrages an treffen ( $\S 88$ Abs. 1 SGB IX). Wird innerhalb dieser Frist eine Entscheidung in den Fällen des $\S 89$ Abs. 1 Satz 1 und Abs. 3 nicht getroffen, gilt die Zustimmung als erteilt (§ 88 Abs. 5). „Diese gesetzliche Fiktion ist die denkbar schärfste Sanktion einer behördlichen Entscheidungsverzögerung, die dem Integrationsamt in der Regel nicht vorgeworfen werden kann"37.

§ 88 Abs. 3 SGB IX schreibt vor, dass wenn das Integrationsamt die Zustimmung zur Kündigung erteilt, der Arbeitgeber die Kündigung nur innerhalb eines Monats nach Zustellung erklären kann. Diese Vorschrift findet auch in den Fällen Anwendung, wenn die Zustimmung als erteilt angenommen wird (Fiktion der Zustimmung, § 88 Abs. 5 Satz 3 SGB IX). „Jede Kündigung, die dem Arbeitnehmer später zugeht, ist unwirksam“38.

Es ist erwähnenswert, dass der deutsche Gesetzgeber das freie Ermessen des Integrationsamtes bei der Erteilung der Zustimmung zur Kündigung begrenzt hat. In § 89 SGB IX wurden die Fälle aufgeführt, in denen das Integrationsamt verpflichtet ist, der Kündigung des Arbeitsverhältnisses eines schwerbehinderten Menschen durch den Arbeitgeber zuzustimmen.

\footnotetext{
35 Lampe, Fußnote 31, S. 72.

36 Ibidem, S. 74.

37 Ibidem, S. 98.

38 R. Rehwald, [in:] W. Feldes, B. Fraunhoffer, R. Rehwald, B. von Seggern, B. Westermann, H. Witt, Schwerbehindertenrecht. Basiskommentar zum SGB IX mit Wahlordnung, Frankfurt am Main: Bund Verlag 2012, S. 259.
} 
Das Integrationsamt erteilt die Zustimmung bei Kündigungen in Betrieben und Dienststellen, die nicht nur vorübergehend eingestellt oder aufgelöst werden, wenn zwischen dem Tage, bis zu dem Gehalt oder Lohn gezahlt wird, mindestens drei Monate liegen. Unter der gleichen Voraussetzung soll es die Zustimmung auch bei Kündigungen in Betrieben und Dienststellen erteilen, die nicht nur vorübergehend wesentlich eingeschränkt werden, wenn die Gesamtzahl der weiterhin beschäftigten schwerbehinderten Menschen zur Erfüllung der Beschäftigungsplicht nach $\S 71$ ausreicht (§ 89 Abs. 1 Satz 1 und 2 SGB IX).

Das Integrationsamt soll die Zustimmung erteilen, wenn dem schwerbehinderten Menschen ein anderer angemessener und zumutbarer Arbeitsplatz gesichert ist (§89 Abs. 2 SGB IX).

Ist das Insolvenzverfahren über das Vermögen des Arbeitgebers eröffnet, soll das Integrationsamt die Zustimmung erteilen, wenn:

- der schwerbehinderte Mensch in einem Interessenausgleich namentlich als einer der zu entlassenden Arbeitnehmer bezeichnet ist (§ 125 der Insolvenzordnung);

- die Schwerbehindertenvertretung beim Zustandekommen des Interessenausgleichs gemäß § 95 Abs. 2 beteiligt worden ist;

- der Anteil der nach dem Interessenausgleich zu entlassenden schwerbehinderten Menschen an der Zahl der beschäftigten schwerbehinderten Menschen nicht größer ist als der Anteil der $\mathrm{zu}$ entlassenden übrigen Arbeitnehmer an der Zahl der beschäftigten übrigen Arbeitnehmer und

- die Gesamtzahl der schwerbehinderten Menschen, die nach dem Interessenausgleich bei dem Arbeitgeber verbleiben sollen, zur Erfüllung der Beschäftigungspflicht nach § 71 ausreicht (§ 89 Abs. 3 SGB IX).

\subsection{EINSCHRÄNKUNGEN DER ZULÄSSIGKEIT DER BEENDIGUNG DES}

ARBEITSVERHÄLTNISSES OHNE KÜNDIGUNG DURCH DEN ARBEITGEBER

„Das Zustimmungsverfahren zur fristlosen Kündigung ist in § 91 SGB IX geregelt. Das Gesetz spricht in § 91 Abs. 1 SGB IX nicht von der fristlosen, sondern von der außerordentlichen Kündigung. Der Begriff 
selbst ist im SGB IX nicht definiert. Es gilt vielmehr die allgemeine Vorschrift des § 626 Abs. 1 BGB“39.

„Auch außerordentliche, fristlose Kündigungen durch den Arbeitgeber bedürfen der vorherigen Zustimmung des Integrationsamtes. Die Vorschriften der $\S \S 85,87,90$ sowie 92 gelten auch für fristlose Kündigungen" 40 . Der Arbeitgeber muss beim zuständigen Integrationsamt den Antrag auf Zustimmung zur Beendigung des Arbeitsverhältnisses mit einem schwerbehinderten Arbeitnehmer ohne Kündigung stellen (§ 87 Abs. 1 in Verbindung mit $\S 91$ Abs. 1 SGB IX). Die Zustimmung zur außerordentlichen Kündigung kann nur innerhalb von zwei Wochen beantragt werden. Die Frist beginnt mit dem Zeitpunkt, in dem der Arbeitgeber von den für die Kündigung maßgebenden Tatsachen Kenntnis erlangt (§91 Abs. 2 SGB IX).

Das Integrationsamt trifft die Entscheidung innerhalb von zwei Wochen vom Tage des Eingangs des Antrages an. Wird innerhalb dieser Frist eine Entscheidung nicht getroffen, gilt die Zustimmung als erteilt (\$ 91 Abs. 3 SBG IX). „Der Arbeitgeber kann dann kündigen"41. Der deutsche Gesetzgeber hat das freie Ermessen des Integrationsamtes bei der Erteilung der Zustimmung zur Beendigung des Arbeitsverhältnisses ohne Kündigung begrenzt. Nach § 91 Abs. 4 SGB IX soll das Integrationsamt die Zustimmung erteilen, wenn die Kündigung aus einem Grund erfolgt, der nicht im Zusammenhang mit der Behinderung steht.

\subsection{Die ROLLE DES INTEGRATIONSAMTES}

Das Integrationsamt soll in jeder Lage des Verfahrens eine gütliche Einigung anstreben ${ }^{42}$. In der einschlägigen Fachliteratur wird hervorgehoben, dass "die ideale gütliche Einigung meistens die Weiterbeschäftigung des schwerbehinderten Menschen beinhalten wird, die das Gesetz [SGB IX] insbesondere durch § 81 verstärkt ermöglichen will und die deshalb auch das Integrationsamt gemäß § 89 Abs. 1 Satz 3

\footnotetext{
39 Besgen, Fußnote 32, S. 89.

40 Rehwald, Fußnote 39, S. 272.

41 Ibidem, S. 274.

42 S. § 87 Abs. 3 SGB IX.
} 
anzustreben hat" 43 . Die Bemühungen des Integrationsamtes um eine gütliche Einigung sollten auf eine Weiterbeschäftigung des schwerbehinderten Mitarbeiters in demselben Betrieb oder auf einem anderen Arbeitsplatz in einem anderen Betrieb desselben Arbeitgebers mit dem Einverständnis des Betroffenen ausgerichtet sein. Außerdem muss die Weiterbeschäftigung des behinderten Arbeitnehmers für den Arbeitgeber zumutbar sein. Somit wird nicht nur das rechtliche Interesse des behinderten Arbeitnehmers, sondern auch das des Arbeitgebers geschützt.

\section{KÜNDIGUNGSSCHUTZ FÜR MENSCHEN MIT BEHINDERUNGEN IM POLNISCHEN RECHT}

\subsection{ALlGemeine BEMERKUnGeN}

Der polnische Gesetzgeber hat expressis verbis anerkannt, dass Menschen mit Behinderungen sich auf dem Arbeitsmarkt in einer besonderen Lage befinden ${ }^{44}$. Er hat Regelungen getroffen, die die Chancen der Menschen mit Behinderungen auf einen Zugang zur Beschäftigung am offenen Arbeitsmarkt ausgleichen sollen ${ }^{45}$. Daneben hat er die Möglichkeit der Beschäftigung in Werkstätten für behinderte Menschen vorgesehen.

Arbeitnehmern mit einer durch zuständige Organe festgestellten Behinderung stehen zusätzliche Rechte $\mathrm{zu}$, insbesondere im Bereich der Arbeitszeit, des zusätzlichen Erholungsurlaubs und zusätzlicher Freistellungen von der Arbeit. Der Arbeitgeber ist dagegen verpflichtet, rationale Anpassungen, die den Bedürfnissen behinderter Menschen Rechnung tragen, im Betrieb zu treffen. In der Fachliteratur wird bemerkt,

\footnotetext{
43 Lampe, Fußnote 31, S. 132.

44 S. Artikel 49 Pkt. 6 des Gesetzes über Beschäftigungsförderung und die Arbeitsmarktinstitutionen vom 20.04.2004, Einheitlicher Text: Dz.U. [Gesetzblatt] von 2015 Pos. 149 m. Änd.

45 Mehr dazu s. M. Szabłowska, Wyrównywanie szans osób niepetnosprawnych w zakresie dostępu do zatrudnienia na otwartym rynku pracy [Ausgleich der Chancen behinderter Menschen auf einen Zugang zur Beschäftigung am offenen Arbeitsmarkt], Polityka Społeczna [Sozialpolitik] 2013, Nr. 10, S. 20 ff.
} 
dass „die Beschäftigung von Menschen mit Behinderungen (...) im Wirkungsbereich der arbeitsrechtlichen Normen liegt. Die Auswirkung der arbeitsrechtlichen Normen bezieht sich hier lediglich auf die Beschäftigungsbedingungen, ein besonderer Kündigungsschutz kommt dagegen nicht zur Anwendung" ${ }^{46}$.

Im Gegensatz zum deutschen Gesetzgeber hat der polnische Gesetzgeber keinen besonderen Kündigungsschutz aus Gründen der Behinderung vorgesehen. Er hat kein Verbot der Kündigung des Arbeitsverhältnisses wegen einer Behinderung, keine Verpflichtung des Arbeitgebers, eine Zustimmung zur Entlassung eines behinderten Arbeitnehmers $\mathrm{zu}$ erlangen, sowie keinen geschlossenen Katalog der Kündigungsgründe im Fall von Menschen mit Behinderungen beschlossen ${ }^{47}$. Er hat lediglich rechtliche Instrumente zur Stabilisierung der Beschäftigung von Menschen mit Behinderungen geschaffen.

\subsection{STABILISIERUNG DER BESCHÄFTIGUNG BEI BEHINDERUNG INFOLGE EINES ARBEITSUNFALLS ODER EINER BERUFSKRANKHEIT}

Als das wesentliche rechtliche Instrument zur Stabilisierung der Beschäftigung von Menschen, deren Behinderung durch einen Arbeitsunfall oder eine Berufskrankheit verursacht wurde, ist die in Artikel 14 des Rehabilitationsgesetzes festgehaltene Verpflichtung des Arbeitgebers anzusehen.

Nach Artikel 14 des Rehabilitationsgesetzes muss der Arbeitgeber für die Person, die infolge eines Arbeitsunfalls oder einer Berufskrankheit unfähig wurde, am bisherigen Arbeitsplatz zu arbeiten, und bei der Behinderung festgestellt wurde, einen angemessenen Arbeitsplatz mit dem dazugehörigen Sozialraum ausgliedern bzw. einrichten, sofern diese Person innerhalb eines Monats nach Feststellung der Behinderung ihre Bereitschaft anmeldet, die Arbeit aufzunehmen. Die Ausgliederung bzw. Einrichtung des Arbeitsplatzes mit dazugehörigem Sozialraum muss innerhalb von drei Monaten nach Anmeldung der Bereitschaft zur

46 M. Skąpski, Ochronna funkcja prawa pracy $w$ społecznej gospodarce rynkowej [Die Schutzfunktion des Arbeitsrechts in der sozialen Marktwirtschaft], Kraków: Kantor Wydawniczy Zakamycze 2006, S. 137-138.

47 Vgl. Latos-Miłkowska, Fußnote 29, S. 263. 
Arbeitsaufnahme erfolgen. „Die Erfüllung der Verpflichtung nach Artikel 14 des Gesetzes über berufliche und soziale Rehabilitation sowie Beschäftigung von Menschen mit Behinderungen wurde nicht davon abhängig gemacht, ob der Arbeitgeber über erforderliche Mittel dazu verfügt. Die Einschränkung (...), dass die zu treffenden Vorkehrungen für den Arbeitnehmer zumutbar sein müssten, ist hier nicht vorhanden. Der Arbeitgeber muss somit alle erforderlichen Maßnahmen ergreifen, um dem behinderten Arbeitnehmer eine weitere Arbeitsleistung am ausgegliederten Arbeitsplatz zu ermöglichen" 48 .

In der Fachliteratur wird zutreffend bemerkt, dass die Regelung des Artikel 14 des Rehabilitationsgesetzes „vorbeugen soll, dass das Arbeitsverhältnis nach Ablauf der während der Arbeitsunfähigkeit, die im Zusammenhang mit Arbeitsunfall oder Berufskrankheit steht, geltenden Schutzfrist beendet wird. Die Entlassungsmöglichkeiten werden nämlich zugunsten der beruflichen Rehabilitation des Arbeitnehmers und seiner Anstellung an einem anderen, seinem Gesundheitszustand und seiner Leistungsfähigkeit angepassten Arbeitsplatz, wesentlich eingeschränkt" 49 . Die Regelung des Artikel 14 des Rehabilitationsgesetzes erleichtert es den Menschen, deren Behinderung während der Beschäftigung eingetreten ist, ihr Recht auf Arbeit zu verwirklichen und wirkt somit der Ausgrenzung dieser Menschen am Arbeitsmarkt entgegen. Dies steht im Einklang mit den Vorschriften des Artikel 27 Abs. 1 des von Polen ratifizierten UN-Übereinkommens vom 13. Dezember 2006 über die Rechte von Menschen mit Behinderungen ${ }^{50}$.

Die Pflicht des Arbeitgebers, einen angemessenen Arbeitsplatz mit dem dazugehörigen Sozialraum auszugliedern bzw. einzurichten, besteht nicht, wenn eine durch den Arbeitgeber bewiesene, schuldhafte oder im Zustand nach Alkoholkonsum begangene Verletzung von Vorschriften des Arbeits- und Gesundheitsschutzes durch den

\footnotetext{
48 Ibidem, S. 261.

49 W. Witoszko, Ochrona trwałości stosunku pracy osób niepetnosprawnych [Kündigungsschutz für Menschen mit Behinderungen], [in:] G. Goździewicz (Red.), Ochrona trwałości stosunku pracy $w$ społecznej gospodarce rynkowej [Der Kündigungsschutz in der sozialen Marktwirtschaft], Warszawa: Wolters Kluwer Polska 2010, S. 430.

50 Dz.U. [Gesetzblatt] von 2012, Pos. 1169.
} 
Arbeitnehmer die ausschließliche Ursache des Arbeitsunfalls war ${ }^{51}$. $\mathrm{Zu}$ beachten ist jedoch, dass selbst dann, wenn eine bewiesene, schuldhafte oder im Zustand nach Alkoholkonsum begangene Verletzung von Vorschriften des Arbeits- und Gesundheitsschutzes durch den Arbeitnehmer die ausschließliche Ursache des Arbeitsunfalls war, der Arbeitgeber nicht von der Pflicht nach Artikel 231 des Arbeitsgesetzbuches befreit ist. Nach Maßgabe dieses Artikels muss der Arbeitgeber einen Arbeitnehmer, der infolge eines Arbeitsunfalls oder einer Berufskrankheit unfähig wurde, am bisherigen Arbeitsplatz zu arbeiten, und der nicht nach dem SVF-Rentengesetz als Erwerbsunfähig anerkannt wurde, an einen angemessenen Arbeitsplatz versetzen. Die Versetzung an einen angemessenen Arbeitsplatz erfolgt aufgrund einer ärztlichen Bescheinigung, die nach Maßgabe der Verordnung des Ministers für Gesundheit und soziale Fürsorge über die arbeitsmedizinischen Vorsorgeuntersuchungen, den Umfang der vorbeugenden Fürsorge und die Bescheinigungen für die im Arbeitsgesetzbuch vorgesehenen Zwecke vom 30. Mai 1996, ausgestellt wird52. Praktisch ist eine Situation denkbar, in der der Arbeitnehmer nicht nach dem SVF-Rentengesetz als voll oder teilweise Erwerbsunfähig anerkannt wurde (Bescheid des ärztlichen Gutachters der Sozialversicherungsanstalt), bei dem aber einer der drei Grade der Behinderung festgestellt wurde (Bescheinigung des Ausschusses für Behinderungsfeststellung). Der Arbeitgeber muss eine solche Person an einen angemessenen Arbeitsplatz versetzen und ihr sechs Monate lang eine Ausgleichszulage zahlen, falls die Versetzung mit der Gehaltsminderung einhergeht. Der Anspruch auf Ausgleichszulage besteht selbst dann, wenn

\footnotetext{
51 S. Artikel 14 Abs. 2 Rehabilitationsgesetz. Die Ausgestaltung dieser Vorschrift verdient eine Anmerkung. Sie unterscheidet sich von der Formulierung der Prämissen für den Ausschluss des Anspruchs auf Unfallversicherungsleistungen in Artikel 21 des Gesetzes über Sozialversicherung bei Arbeitsunfällen und Berufskrankheiten vom 30.10.2002, einheitlicher Text: Dz.U. [Gesetzblatt] von 2009, Nr. 167, Pos. 1322 m. Änd. Darüber hinaus wurde in Artikel 14 Abs. 2 des Rehabilitationsgesetzes, anders als etwa in Artikel 21 des vorgenannten Gesetzes, neben dem "Zustand nach Alkoholkonsum" nicht der "Zustand nach Konsum von Betäubungsmitteln oder psychotropen Substanzen“ erwähnt. Es erscheint als angebracht, in den Artikel 14 Abs. 2 des Rehabilitationsgesetzes eine angemessene Ergänzung einzubauen, damit keine Zweifel daran aufkommen, dass der Gesetzgeber den Zustand nach Konsum von Betäubungsmitteln oder psychotropen Substanzen mit dem Zustand nach Alkoholkonsum gleich behandelt.
}

52 Dz.U. [Gesetzblatt] Nr. 69, Pos. 332 m. Änd., 
eine bewiesene, schuldhafte oder im Zustand nach Alkoholkonsum begangene Verletzung von Vorschriften des Arbeits- und Gesundheitsschutzes durch den Arbeitnehmer die ausschließliche Ursache des Arbeitsunfalls war ${ }^{53}$.

Über die in Artikel 14 des Rehabilitationsgesetzes formulierte Verpflichtung des Arbeitgebers hinaus, einen angemessenen Arbeitsplatz mit dem dazugehörigen Sozialraum auszugliedern bzw. einzurichten, führte der polnische Gesetzgeber eine Geldstrafe für Arbeitgeber ein, die dieser Verpflichtung innerhalb der vorgeschriebenen Frist nicht nachgehen ${ }^{54}$. Die Geldstrafe entspricht dem fünfzehnfachen durchschnittlichen Monatsgehalt und ist an den Staatlichen Fonds für die Rehabilitation von Menschen mit Behinderungen zu zahlen. Die Zahlung an den Staatlichen Fonds für die Rehabilitation von Menschen mit Behinderungen hat am Tag der Beendigung des Arbeitsverhältnisses mit der Person zu erfolgen, für die kein angemessener Arbeitsplatz mit dem dazugehörigen Sozialraum ausgegliedert wurde (Artikel 23 Rehabilitationsgesetz). Es ist erwähnenswert, dass das früher geltende Gesetz über Beschäftigung und berufliche Rehabilitation von Menschen mit Behinderungen vom 9. Mai $1991^{55}$ in Artikel 6 Abs. 2 eine Zahlung an den Staatlichen Fonds für die Rehabilitation von Menschen mit Behinderungen in Höhe des vierzigfachen durchschnittlichen Monatsgehalts vorschrieb.

Nicht zu übersehen ist auch, dass weder im derzeit geltenden Gesetz über berufliche und soziale Rehabilitation sowie Beschäftigung von Menschen mit Behinderungen von 1997 noch im früheren Gesetz über Beschäftigung und berufliche Rehabilitation von Menschen mit Behinderungen von 1991 Schadensersatzleistungen für den Arbeitnehmer vorgesehen wurden, für den der Arbeitgeber keinen angemessenen Arbeitsplatz mit dem dazugehörigen Sozialraum ausgegliedert bzw. eingerichtet hat. Es hat den Anschein, dass legislative Bemühungen zur Einführung einer Schadensersatzpflicht des Arbeitgebers gegenüber dem Arbeitnehmer im Fall der Nichterfüllung der Verpflichtung nach Artikel 14 des Rehabilitationsgesetzes durchaus angezeigt wären.

53 Vgl. A. Landkamer-Prasołek, [in:] K. Walczak (Red.), Komentarz do Kodeksu pracy [Kommentar zum Arbeitsgesetzbuch], Warszawa: C.H. Beck 2012, S. 209.

54 Vgl. Latos-Miłkowska, Fußnote 29, S. 262.

55 Dz.U. [Gesetzblatt] Nr. 46, Pos. 201 m. Änd. 
Es ist außerdem festzuhalten, dass gegenüber einem Arbeitnehmer, dessen Behinderung auf einen Arbeitsunfall oder eine Berufskrankheit zurückzuführen ist, der Arbeitgeber neben der Pflicht nach Artikel 14 des Rehabilitationsgesetzes auch noch die Pflicht zur Einführung rationaler Anpassungen hat (Artikel 23a Rehabilitationsgesetz). Wird die letztere nicht erfüllt, so kann der Arbeitnehmer vom Arbeitgeber Schadensersatz wegen Verletzung des Gleichbehandlungsgrundsatzes bei der Beschäftigung verlangen. Praktisch ist es möglich, dass sowohl die Pflicht nach Artikel 14 als auch die nach Artikel 23a des Rehabilitationsgesetzes zugleich verletzt werden. Der Arbeitgeber wäre dann zur Zahlung an den Staatlichen Fonds für die Rehabilitation von Menschen mit Behinderungen verpflichtet. Außerdem könnte der Arbeitnehmer gegen ihn Schadensersatz wegen Verletzung des Gleichbehandlungsgrundsatzes bei der Beschäftigung geltend machen.

\subsection{StABILISIERUNG DER BESCHÄFTIGUNG IM FALL EINER BEHINDERUNG INFOLGE EINES UNFALLS AUF DEM WEG ZUR ARBEIT ODER EINES NICHT IM ZUSAMMENHANG MIT DER ARBEIT STEHENDEN EREIGNISSES}

Gegenüber den Arbeitnehmern, die ihre Fähigkeit zur Arbeitsleistung am bisherigen Arbeitsplatz verloren haben und als Menschen mit Behinderung anerkannt wurden, wobei die Ursache für die Behinderung ein Unfall auf dem Weg von oder zur Arbeit bzw. ein anderes nicht im Zusammenhang mit der Arbeit stehendes Ereignis war, ist der Arbeitgeber nicht nach Artikel 14 des Rehabilitationsgesetzes verpflichtet. Der Arbeitgeber muss aber notwendige rationale Anpassungen an die Bedürfnisse dieser Arbeitnehmer vornehmen, indem er im gegebenen Fall erforderliche Änderungen bzw. Optimierungen einführt, die den besonderen, ihm gemeldeten Bedürfnissen des behinderten Arbeitnehmers Rechnung tragen. Der Arbeitgeber ist von dieser Pflicht befreit, wenn die Einführung notwendiger rationaler Anpassungen unverhältnismäßige Belastungen für ihn nach sich ziehen würde. Werden die vom Arbeitnehmer zu tragenden Belastungen aus den öffentlichen Mitteln im ausreichenden Maße erstattet, gelten sie nicht als unverhältnismäßig (Artikel 23a Abs. 1-2 Rehabilitationsgesetz). Der Arbeitgeber, der rationale Anpassungen an die Bedürfnisse der Arbeitnehmer vornimmt, deren 
Behinderung während der Dauer des Arbeitsverhältnisses eingetreten ist, kann eine Erstattung der dafür aufgewendeten Kosten aus dem Staatlichen Fonds für die Rehabilitation von Menschen mit Behinderungen erlangen (Artikel 26 Abs. 2 Pkt. 2 Rehabilitationsgesetz). Die Erstattung umfasst die Kosten der Anpassung der Betriebsräumlichkeiten an die Bedürfnisse der Menschen mit Behinderungen, der Anpassung bzw. des Erwerbs von Geräten, die den Menschen mit Behinderungen die Arbeitsleistung bzw. die Bewegung im Betrieb ermöglichen, die Anschaffung und Autorisierung der Software für behinderte Arbeitnehmer sowie der an ihre Bedürfnisse angepassten Hilfsgeräte und technologien sowie die Kosten der Feststellung notwendiger Änderungen und Anpassungen im gegebenen Fall durch arbeitsmedizinisches Personal (Artikel 26 Abs. 1 Rehabilitationsgesetz). Nimmt der Arbeitgeber die notwendigen rationalen Anpassungen aber nicht vor, trifft ihn Schadensersatzhaftung wegen Verletzung des Gleichbehandlungsgrundsatzes bei der Beschäftigung nach Artikel 183d des Arbeitsgesetzbuches.

Die Einführung notwendiger rationaler Anpassungen an die Bedürfnisse der Arbeitnehmer, deren Behinderung während der Dauer des Arbeitsverhältnisses eingetreten ist, ermöglicht eine Weiterbeschäftigung dieser Arbeitnehmer. In der Fachliteratur wird es zutreffend als „Maßnahme zur Stabilisierung des Arbeitsverhältnisses des behinderten Arbeitnehmers" 56 bezeichnet. Zur Stabilisierung des Arbeitsverhältnisses tragen ebenfalls die vom Gesetzgeber beschlossenen Regeln zur Erstattung zusätzlicher Kosten, die der Arbeitgeber im Zusammenhang mit der Beschäftigung von Menschen mit Behinderungen getragen hat. Die Kosten werden dem Arbeitgeber aus dem Staatlichen Fonds für die Rehabilitation von Menschen mit Behinderungen erstattet, sofern der behinderte Arbeitnehmer für mindestens 36 Monate beschäftigt wird. Sollte der Beschäftigungszeitrum kürzer als 36 Monate sein, muss der Arbeitgeber unter Vermittlung des Landrates [poln. starosta] eine Rückerstattung in Höhe von 1/36 pro fehlenden Monat, mindestens aber 1/ 6 dieses Betrages, an den Staatlichen Fonds für die Rehabilitation von Menschen mit Behinderungen leisten. Der Arbeitgeber hat die Zahlung innerhalb von

56 Latos-Miłkowska, Fußnote 29, S. 259. 
3 Monaten nach Beendigung des Arbeitsverhältnisses mit dem behinderten Arbeitnehmer zu leisten ${ }^{57}$.

\subsection{BERUFLICHE REHABILITATION ALS INSTRUMENT ZUR STABILISIERUNG DER BESCHÄFTIGUNG}

Ein wirksames System der beruflichen Rehabilitation kann einen wesentlichen Beitrag zur Stabilisierung der Beschäftigung von Menschen mit Behinderungen leisten.

Nach Artikel 8 Abs. 1 des Rehabilitationsgesetzes soll die berufliche Rehabilitation dem Menschen mit Behinderung helfen, durch Zugang zur Berufsberatung, Ausbildung und Arbeitsvermittlung eine Beschäftigung und den beruflichen Aufstieg $\mathrm{zu}$ finden und $\mathrm{zu}$ behalten. Berufliche Rehabilitation kann somit wirksam der Ausgrenzung der Personen am Arbeitsmarkt entgegenwirken, deren Behinderung während der Dauer des Arbeitsverhältnisses eingetreten ist. $\mathrm{Zu}$ begrüßen ist insbesondere die Förderung aus öffentlichen Mitteln der Rehabilitation von Menschen mit Behinderungen, die auf ausgegliederten Arbeitsplätzen bei Arbeitnehmern des offenen Arbeitsmarktes beschäftigt werden.

In Polen funktionieren zwei separate Systeme der beruflichen Rehabilitation: das System der beruflichen Rehabilitation von Menschen mit Behinderungen und das System der beruflichen Rehabilitation erwerbsgeminderter Arbeitnehmer. Es ist $\mathrm{zu}$ beachten, dass sich unter den Erwerbsgeminderten auch Personen befinden können, deren Behinderung mit einem entsprechenden Bescheid festgestellt wurde. Das Gesetz über berufliche und soziale Rehabilitation sowie Beschäftigung von Menschen mit Behinderungen vom 27. August 199758 sieht drei Organisationsformen vor, auf die das System zur beruflichen Rehabilitation behinderter Menschen gestützt ist: Werkstätten für Ergotherapie, Werkstätten für berufliche Aktivität und geschützte Werkstätten ${ }^{59}$.

\footnotetext{
57 S. Artikel 26 Abs. 1 und Artikel 26 Abs. 7 Rehabilitationsgesetz.

58 Einheitlicher Text: Dz.U. [Gesetzblatt] von 2011, Nr. 127, Pos. 721 m. Änd.

59 Mehr dazu s. A. Barczyński, Propozycje kierunku modyfikacji systemu rehabilitacji zawodowej osób niepetnosprawnych [Vorschläge zur Modifizierung des Systems beruflicher Rehabilitation von Menschen mit Behinderungen], [in:] Polskie doświadczenia w rehabilitacji zawodowej $i$ zatrudnianiu osób niepetnosprawnych. Referaty [Polnische Erfahrungen mit der beruflichen
} 
Gem. \& 3 der Verordnung des Ministerrates über die Bedingungen der Teilnahme an einer zum Übergangsgeld berechtigenden beruflichen Rehabilitation und über detaillierte Regeln zum Erwerb des Anspruchs auf Übergangsgeld vom 27. Dezember $1974^{60}$ können erwerbsgeminderte Arbeitnehmer einer beruflichen Rehabilitation in betrieblichen und betriebsübergreifenden Zentren für berufliche Rehabilitation sowie an ausgegliederten Arbeitsplätzen unterzogen werden. Wie bereits erwähnt, kann die Erwerbsminderung von einer mit entsprechendem Bescheid festgestellten Behinderung begleitet sein. Im Fall der Arbeitnehmer, deren Erwerbsminderung bzw. Behinderung während der Dauer des Arbeitsverhältnisses eingetreten sind, würde eine Unterstützung und Förderung der beruflichen Rehabilitation am ausgegliederten Arbeitsplatz beim bisherigen Arbeitgeber aus öffentlichen Mitteln als durchaus wünschenswert erscheinen61. Dieses Problem wird aber nicht ausreichend beachtet.

\section{FAZIT}

$\mathrm{Zu}$ den rechtlichen Instrumenten des Kündigungsschutzes für Menschen mit Behinderungen gehören rechtliche Maßnahmen zum besonderen Kündigungsschutz sowie Maßnahmen zur Stabilisierung des Arbeitsverhältnisses, darunter das Verbot der Diskriminierung wegen einer Behinderung bei der Beendigung des Arbeitsverhältnisses, die Pflicht zur Einführung rationaler Anpassungen an die Bedürfnisse der Menschen mit Behinderungen, die Pflicht zur Ausgliederung bzw. Einrichtung eines angemessenen Arbeitsplatzes mit dem dazugehörigen Sozialraum für den Arbeitnehmer, dessen Behinderung während der Dauer des Arbeitsverhältnisses eingetreten und auf einen Arbeitsunfall oder eine Berufskrankheit zurückzuführen ist.

Rehabilitation und der Beschäftigung von Menschen mit Behinderungen. Beiträge], Warszawa: Krajowa Izba Gospodarczo-Rehabilitacyjna 2003, S. 165.

60 Dz.U. [Gesetzblatt] Nr. 51, Pos. 325 m. Änd.

61 S. auch: M. Szabłowska-Juckiewicz, Prawo do pracy osób niepetnosprawnych - zarys problematyki [Das Recht der Menschen mit Behinderungen auf Arbeit - ein Leitfaden], A. Giedrewicz-Niewińska, M. Szabłowska-Juckiewicz (Red.), Zatrudnianie osób niepetnosprawnych. Regulacje prawne [Beschäftigung von behinderten Menschen. Rechtliche Regelungen], Warszawa: Difin 2014, S. 74. 
Die EU-Vorgaben zur Gleichbehandlung in Beschäftigung und Beruf hatten einen wesentlichen Einfluss auf die rechtlichen Regelungen, die von dem deutschen und dem polnischen Gesetzgeber getroffen wurden. Behinderung zählt zu den Eigenschaften, die dem Diskriminierungsverbot unterliegen. Der sachliche Anwendungsbereich des Diskriminierungsverbotes wegen Behinderung in Beschäftigung und Beruf ist weit gefasst. Dies gilt insbesondere für die Beendigung des Arbeitsverhältnisses. Folglich kann Behinderung, unabhängig von deren Grad und davon, ob sie vor oder während der Dauer des Arbeitsverhältnisses eingetreten ist, nicht Kündigungsgrund sein.

Auf die Einführung des Verbotes der Diskriminierung in Beschäftigung und Beruf wegen der Behinderung, darunter in Bezug auf Beendigung des Arbeitsverhältnisses, folgt die Festlegung der Pflichten des Arbeitgebers im Hinblick auf im Betrieb vorzunehmende Anpassungen an die Bedürfnisse behinderter Menschen, unabhängig davon, ob die Behinderung vor oder während der Dauer des Arbeitsverhältnisses eingetreten ist. Es ist darauf hinzuweisen, dass die besonderen Pflichten des Arbeitgebers in Bezug auf die Anpassung des Arbeitsplatzes an die Bedürfnisse des behinderten Arbeitnehmers bzw. auf die Versetzung an einen anderen, passenden Arbeitsplatz dann zur Geltung kommen, wenn die Behinderung auf einen Arbeitsunfall oder eine Berufskrankheit zurückzuführen ist.

Der deutsche Gesetzgeber beschloss Regelungen zum besonderen Kündigungsschutz für schwerbehinderte Menschen. Hervorzuheben ist, dass der besondere Kündigungsschutz nicht bei allen behinderten Arbeitnehmern, sondern nur bei den schwerbehinderten zur Anwendung kommt. Als rechtliches Instrument zur Verwirklichung des besonderen Kündigungsschutzes dient die Verpflichtung des Arbeitgebers, vor der Kündigung bzw. der Beendigung des Arbeitsverhältnisses ohne Kündigung eine Zustimmung des Integrationsamtes einzuholen.

Der polnische Gesetzgeber hat sich nicht dazu entschieden, Menschen mit Behinderungen unter einen besonderen Kündigungsschutz zu stellen. Er hat aber andere rechtliche Maßnahmen beschlossen, die zur Stabilisierung der Beschäftigung behinderter Menschen beitragen sollen. Dazu gehören finanzielle Anreize für Arbeitgeber, die Menschen mit Behinderungen beschäftigen, ebenso wie finanzielle Sanktionen für 
diejenigen, die auf behinderte Arbeitnehmer und auf Maßnahmen zur Einrichtung eines an ihre Bedürfnisse angepassten Arbeitsplatzes bzw. zur Anpassung des bisherigen Arbeitsplatzes verzichten, wenn die Behinderung während der Dauer des Arbeitsverhältnisses eingetreten und auf einen Arbeitsunfall oder eine Berufskrankheit zurückzuführen ist ${ }^{62}$. Das System finanzieller Anreize und finanzieller Sanktionen, das großenteils auf der Verpflichtung zu Leistungen an den Staatlichen Fonds für die Rehabilitation von Menschen mit Behinderungen und auf der Befreiung von dieser Verpflichtung beruht, erscheint als unzureichend. Die Wirksamkeit der beruflichen Rehabilitation der Menschen mit Behinderungen, die zur Stabilisierung ihrer Beschäftigung beitragen könnte, ist ebenso wenig zufriedenstellend. Als unentbehrlich erscheinen daher die Änderungen im Organisationsrahmen für die berufliche Rehabilitation der Menschen mit Behinderungen, insbesondere durch eine Aufwertung der beruflichen Rehabilitation am ausgegliederten Arbeitsplatz beim bisherigen Arbeitgeber im Falle von Personen, deren Behinderung während der Dauer des Arbeitsverhältnisses eingetreten ist. Erwägenswert wäre ggf. auch ein besonderer Kündigungsschutz für einige Kategorien behinderter Menschen, der durch die Verpflichtung des Arbeitgebers zur Einholung einer Zustimmung des Integrationsamtes vor der Kündigung bzw. der Beendigung des Arbeitsverhältnisses ohne Kündigung verwirklicht würde.

62 Vgl. T. Wyka, Ochrona zdrowia i życia pracownika jako element treści stosunku pracy [Schutz der Gesundheit und des Lebens des Arbeitnehmers als inhaltlicher Bestandteil des Arbeitsverhältnisses], Warszawa: Difin 2003, S. 158. 
